ORIGINAL RESEARCH PAPER

A PROSPECTIVE STUDY ON FUNCTIONAL
OUTCOIME IN POSTERIOR CRUCIATE
RETAINING VERSUS POSTERIOR CRUCIATE
SACRIFICING TOTAL KNEE ARTHROPLASTY

Orthopaedics

KEY WORDS: Total knee arthroplasty, posterior cruciate ligament, Knee society knee score, Functional knee score, Womac score.
Dr.Dinesh Mishra* Prof. Dr.Sunil Kumar Dash
Junior Resident, Department of Orthopaedics. Hi-Tech Medical College and Hospital, Bhubaneswar, Odisha *Corresponding Author

HOD, Department of Orthopaedics. Hi-Tech Medical College and Hospital, Bhubaneswar, Odisha.

Background: Total knee arthroplasty has evolved over the past decades into a very much reliable surgical treatment for advanced arthritis of knee. Total knee replacement has been shown to restore patient function and relieve pain and deformity that results from knee arthritis. The success of a total knee replacement is determined by many factors like degree of pain relief and functional outcome. The role of Posterior cruciate ligament in total knee replacement is controversial. The first is to retain the ligament and to preserve as much as possible of the normal anatomy and function of the knee. Preservation of the ligament is thought to enhance stability, femoral rollback, mechanical advantage of the quadriceps muscle and proprioceptive properties. Theoretically it has been suggested that PCL retaining can increases the range of flexion and prevents posterior translation which reduces loosening and excessive polyethylene wear by decreasing the shear stresses at the fixation surfaces. We conducted a prospective study to compare resection with retention of PCL using a standard PCL-retaining cemented total knee replacement and assessed the functional outcome using functional knee scores andWOMAC score.

Aims and Objectives: The aim of the study is to "prospectively compare the functional outcome between posterior cruciate ligament retaining and posterior cruciate ligament sacrificing total knee arthroplasty.

Materials and Methods: The study was done on 20 patients admitted to Hi-Tech Medical College \& Hospital, Bhubaneswar. Scoring system formulated by the WOMAC Score, Knee Society Knee Score and Functional Knee Score were used to evaluate the patients before and after surgery. Both knee scores and functional scores are calculated with each amounting to a total of 100 points and WOMAC Score with max of 96 points. All patients were evaluated postoperatively and followed up.

Results: Analyzing the functional outcome it was found that all the patients in both the groups had significant improvement in their knee score and the functional knee score. Analyzing the total Knee Scores, the average Knee Society Score for the PS group was 85.80 and that of CR group was 75.60 and statistical analysis revealed a significant difference in the the p-value in favour of Cruciate Sacrificing Prosthesis signifying that Cruciate Sacrificing Prosthesis has better functional outcome. The functional knee society also showed a marked improvement in all patients, for CS group FKS was 99.6 and for CR group it was 91.6.The WOMAC Score also showed a marked improvement. In CS groups it was 24.6 and in CR it was 27.4. Statistical analysis showed a highly significant difference in favor of cruciate sacrificing prosthesis.

Conclusions:Total Knee Arthroplasty in patients in whom posterior cruciate

ligament was sacrificed was found to have a better functional outcome as compared to the retaining group, which can be mainly attributed to the persistence of flexion deformity in cruciate retaining group. In Indian scenario where knee replacement is done at a late stage of osteoarthritis, sacrificing the contracted posterior cruciate ligament has better outcomes as compared to retaining it.

\section{I.INTRODUCTION :}

Total knee arthroplasty has evolved over the past decades into a very much reliable surgical treatment for advanced arthritis of knee. Total knee replacement has been shown to restore patient function and relieve pain and deformity that results from knee arthritis. The success of a total knee replacement is determined by many factors including pain relief, functional outcome, and range of motion. [1] There have been numerous changes in the total knee arthroplasty, like alterations in component geometry, understanding of the rotational alignment of the components, sizing options and modularity, accurate instrumentation for correcting deformity and improved cementation techniques. The best knee replacement is one, which the kinematics of the normal knee is reproduced. Amongst several factors affecting the kinematics, variations in surface geometry and the retention or sacrificing the posterior cruciate ligament is considered especially important. There are four options available to the surgeon.

The first is to retain the ligament and to preserve as much as possible of the normal anatomy and function of the knee. Preservation of the ligament is thought to enhance stability, femoral rollback, mechanical advantage of the quadriceps muscle and proprioceptive properties.[2,3,4] The second option is to excise the ligament in order to facilitate the correction of any fixed deformities.[5] This allows more accurate and reliable soft tissue balancing. The third option is to substitute the ligament with a posterior stabilized tibial insert having a central post, which can engage on a femoral cam during flexion, mimic femoral rollback and reproduce near normal kinematic profiles. [6] The central post may also allow some stability in the antero-posterior plane and act as a secondary stabilizer to a varus or valgus stress. [7] The fourth option is to release the ligament; this offers a compromise between preservation and excision. [8]

\section{METHODOLOGY: \\ MATERIALS AND METHODS}

Place Of Study: Hi-Tech Medical College and Hospital, Bhubaneswar

Period Of Study: November 2019-October 2021

Consent: Written informed consent of the patients was obtained

\section{Inclusion Criteria:}

In our hospital total knee arthroplasty is being done for osteoarthritis, post-traumatic arthritis and rheumatoid arthritis.

This includes varus as well as valgus knees.

1. Osteoarthritis \& Rheumatoid arthritis

2. Age $>40$ yrs

3. Gender-Both 


\section{Kellgren and Lawrence score Grade 3 and 4}

\section{Exclusion Criteria}

1. Age $<40$ yrs

2. Minimal degenerative changes (KL I \& II)

3. Poor skin conditions

4. Varicose veins

5. Medically unfit

\section{STUDY DESIGN: Prospective study.}

After obtaining clearance and approval from the institutional ethical committee and patients fulfilling the predetermined inclusion \& exclusion criteria, was included in the study after obtaining informed consent. During this study period 26 knees were replaced in 18 patients. All patients were followed at 3weeks, 6weeks, 12weeks, and thereafter every 6 months. Among these patients Osteoarthritis (18), Rheumatoid arthritis (06) Posttraumatic arthritis (02). For SIDE Number Right (05) Left (05) Bilateral (08). For Type of Deformity and Number-Varus (20) Valgus (06).

Preoperatively height and weight of the patients recorded. Scoring system formulated by the American knee society used to evaluate the patients before and after surgery. Both knee scores and functional scores calculated with each mounting to a total of 100 points.[9] Preoperative weight bearing radiograph taken to all patients who underwent knee replacement surgery. Radiological grading system [10]as advocated by Kellgren and Lawrence used to evaluate the severity of arthritis and graded from I to IV as follows.

Grade-I:(Doubtful)-Minute osteophytes, doubtful significance

Grade-II:(Mild)-Definite osteophytes, unimpaired joint space

\section{Grade-III:(Moderate)-Moderate diminution of joint space}

Grade-IV: (Severe)- Joint space greatly impaired with sclerosis of subchondral bone

All 18 cases were undergone surgery at various periods of time during the study period. Posterior cruciate retained in 14 knees and sacrificed in 12 knees. In retained cases, congruent poly used as insert and in sacrificed cases, ultra congruent poly used.

Standard post-operative protocol followed as advised by the American knee society. Patients discharged after suture removal on the $12^{\text {th }}$ postoperative day. Regular follow up done at 3 weeks, 6weeks, 12 weeks and then every 6 months. Post operatively patients functional outcome studied using knee society scores.

\section{Statistical Analysis:}

Descriptive and inferential statistical analysis has been carried out in the present study. Significance is assessed at 5 $\%$ level of significance. Chi-square/ Fisher Exact test has been used to find the significance of study.

\section{III.RESULTS:}

A] Age Distribution: The age of the patients who underwent total knee arthroplasty in our series ranged from 47 to 77 years; average was 60.11 years. More than $50 \%$ of the patients belong to sixth decade.

B] Height: The range in our series was from $150 \mathrm{cms}$ to $174 \mathrm{cms}$. The mean was $158.1 \mathrm{lcms}$.

C] Weight: The weight of the patients ranges from 48kgs to $80 \mathrm{kgs}$. The average Weight was $60.03 \mathrm{kgs}$.
D] KNEE SOCIETY SCORE: All patients evaluated by scoring system proposed by The American Knee society. The average preoperative knee society score was 46.73.The average preoperative functional score was 48.46 .

\begin{tabular}{|l|l|l|l|}
\hline & & $\begin{array}{l}\text { KNEE } \\
\text { SCORE }\end{array}$ & $\begin{array}{l}\text { FUNCTIONAL } \\
\text { SCORE }\end{array}$ \\
\hline & Preoperative & 46.73 & 48.46 \\
\hline Postoperative & Cruciate retained & 93.71 & 85.28 \\
\cline { 2 - 4 } & Cruciate sacrificed & 90.83 & 71.66 \\
\hline
\end{tabular}

Of the 20 patients entered into the study, 18 (26 total knee replacements) patients were available for review. Their mean age was 60 years. Total knee arthroplasty was performed on 18 knees for osteoarthritis and 4 for rheumatoid arthritis and 2 for posttraumatic arthritis. 20 varus and 6 valgus knees.Two patients lost follow-up.

The mean pain score, range of movement, knee score , function score between the cruciate retained and the sacrificed groups shows that mean pain score for the retained group was 48.92 and 47.08 for the sacrificed group.

\begin{tabular}{|l|l|}
\hline Group & Mean pain score \\
\hline Cruciate retained & 48.92 \\
\hline Cruciate sacrificed & 47.08 \\
\hline
\end{tabular}

The range of movement for the retained group was 105 degrees and 100 degrees in sacrificed group.

\begin{tabular}{|l|l|}
\hline Group & Mean of movement \\
\hline Retained & 105 degrees \\
\hline Sacrificed & 100 degrees \\
\hline
\end{tabular}

Mean knee society score for retained group is 94.07 and 92.08 for Sacrificed group.

\begin{tabular}{|l|l|}
\hline Group & Mean knee society score \\
\hline Retained & 94.07 \\
\hline Sacrificed & 92.08 \\
\hline
\end{tabular}

Stability assessed in both the antero-posterior and mediolateral planes. The cruciate retained knees were more stable with $83 \%$ ( 16 cases) having less than $5 \mathrm{~mm}$ of antero-posterior tibial translation and $100 \%$ having less than 5 degree of tibial tilt in medio-lateral plane. $75 \%$ (14 cases) of excised group had less than $5 \mathrm{~mm}$ of tibial translation. This laxity not reflected in medio-lateral planes.

\begin{tabular}{|l|l|l|}
\hline Knee & $\begin{array}{l}\text { Normal } \\
\text { Antero-posterior stability }\end{array}$ & $\begin{array}{l}\text { Normal } \\
\text { Medio-lateral } \\
\text { stability }\end{array}$ \\
\hline Retained & $83 \%$ & $100 \%$ \\
\hline Sacrificed & $75 \%$ & $100 \%$ \\
\hline
\end{tabular}

\section{IV.DISCUSSION:}

Total knee arthroplasty for arthritic patients in whom all the conservative measures are exhausted, is an excellent procedure if proper attention paid to the patient selection. As total knee arthroplasty is a surface replacement within the existing soft tissue sleeve, it functions within normal anatomic and physiologic boundaries. Recent information on the outcome of minimally invasive procedures suggests the reduction of the surgical trauma offers early improvement and faster rehabilitation. This effect levels off after 3 months to a result similar to that in patients who had a standard exposure. This means factors other than the exposure and extensor mechanism violation are involved in the reduced functionality after total knee arthroplasty.

Various factors are associated with the onset and progression of osteoarthritis.[11-17].These include genetic factors, age, sex, obesity, occupation, abnormal loading of the joint in kneeling, squatting and cross-legged sitting. The mean age of our patients who had osteoarthritis is lesser than the data available from the western population. The earlier onset of osteoarthritis in individuals with normal range of body mass index explained by the habit of kneeling, squatting, cross- 
legged sitting practiced by the population in this part of the world.

Out of 18 patients 11 had complete obliteration of joint space at the time of presentation, due to lack of awareness about nature of the disease and about the availability of the various treatment modalities including surgery. Low socioeconomic status and illiteracy may be contributing factors for this.

Retention of posterior cruciate ligament in total knee arthroplasty, advocated as a way to transmit load through the ligament to the tibia, to encourage femoral component rollback to increase flexion, and maintaining the joint line. Retention of posterior cruciate ligament results in a central contact area of the femur on the tibia that helps to distribute load evenly on the tibial component.

In our study flexion and standing view radiographs taken postoperatively for all patients. PCL retained cases exhibits femoral rollback when compared to the PCL sacrificed knees. In $99 \%$ of the virgin arthritic knees requiring arthroplasty including rheumatoids posterior cruciate ligament was found to be intact.The intact PCL may have to sacrificed in rare instances. In the knee with severe angular deformity requiring an extensive release on the concave side of the deformity, the intact posterior cruciate ligament can act as a tether and hinder proper balancing of medial and lateral structures. In our experience, this has occurred twice in the last 12 knees. Each knee has presented with angular deformity of 30 degrees and required extensive medial and lateral release.

We have used the scoring system as advocated by the American knee society. According to this system only three main parameters pain, stability, range of motion judged. Flexion contracture, extension lag and misalignment dealt with as deductions. Thus, 100 points given to knee with no pain,125 degrees of motion and less than $5 \mathrm{~mm}$ of anteroposterior and 5 degrees of mediolateral instability.

Functional score considers walking distance and stair climbing with deductions for walking aids. The maximum functional score 100 is given to patients who can walk unlimited distance and go up and down stairs normally. Although some advocate retaining the posterior cruciate ligament in all patients and others argue for posterior cruciate ligament sacrifice and substitution in all patients Laksin et al suggest a more appropriate approach in which implant design selection based on an individual's pathologic criteria.

In our study, posterior cruciate ligament sacrifice was done in patients who had severe end stage degenerative arthritis, valgus and varus deformities of more than 25 degrees, where surgical exposure is challenging and balancing soft tissue is difficult.

All 18 patients evaluated preoperatively and postoperatively using knee society score. Statistically no significant differences in the follow-up mean pain score and mean knee society score observed in both the cruciate retained and sacrificed groups. Anteroposterior and mediolateral instability does not show any significant differences in both the groups. Translation of the proximal tibia posteriorly in flexed knee is very well obvious radiologicaly in sacrificed group indicating posterior cruciate ligament's function as a restraint to translational displacement. Significantly, greater improvement in flexion from preoperative to most recent follow-up assessment seen in patients in the posterior cruciate retaining group compared to the sacrificed group. This is due to femoral roll back defined as the posterior shift of the tibiofemoral contact areas well exhibited radiologicaly after flexing the knee. In addition, a significantly greater improvement in stair climbing and the mean functional score in cruciate retained arthroplasty groups.

Charles Engh has observed that before any technique is to be adopted or recommended there must be a minimum follow up of ten years. Ours is a small series with maximum follow up of only two years, we can't draw any conclusion from our findings.But the average age of our total knee arthroplasty patients is less when compared toWestern literature, the need of revision will be more. Hence by preserving the posterior cruciate ligament and the bone stock the subsequent revision will be easier.

\section{Vi. Case Illustration:}

Tourniquet

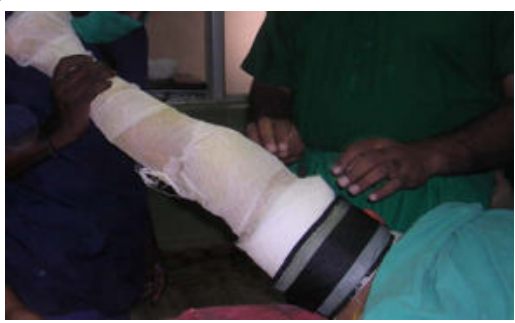

Position

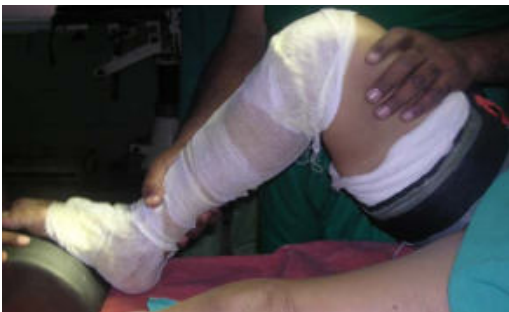

Patella Everted Laterally

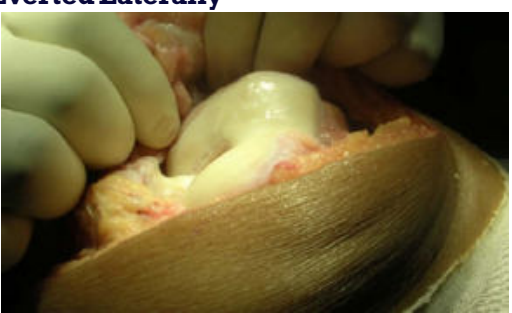

Extramedullary Alignment Jig For Tibia

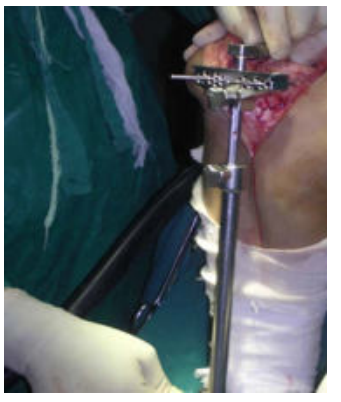

Tibial Cut Made

Intact Pcl After Tibial Cut

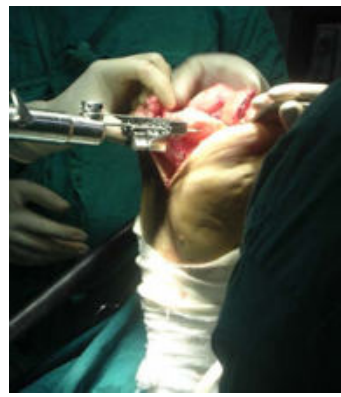

Intramedullary Alignment Made For Femur




Distal Cut Made

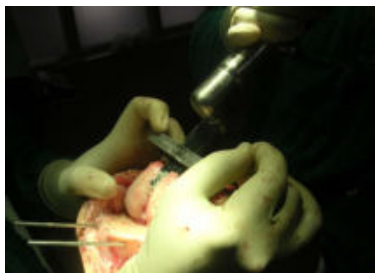

Intact Pcl After

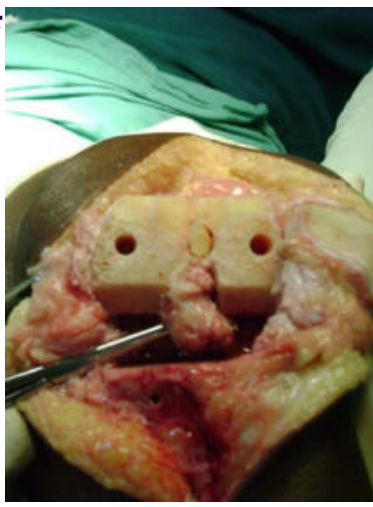

Tibial Implantation After Femoral Implantation After Cementation

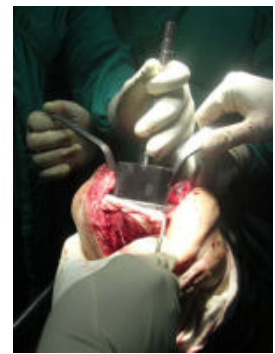

Trial Poly In Place
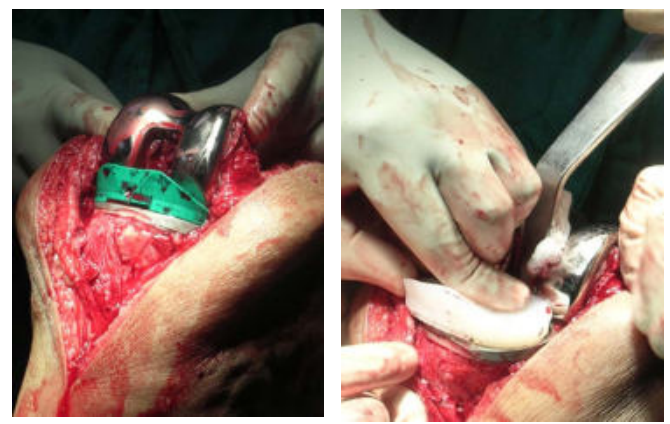

Case (pcl Retained)

Preop Standing



42

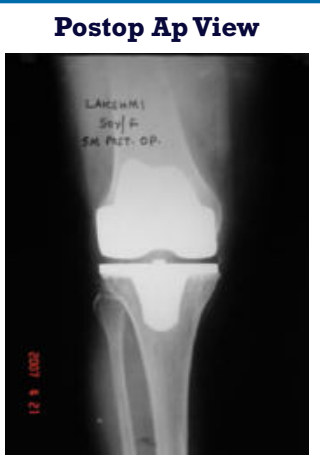

Postop Flexion

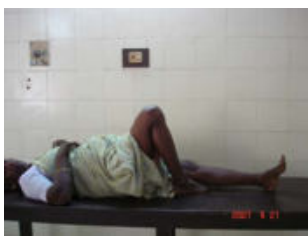

Postop Flexion X-ray

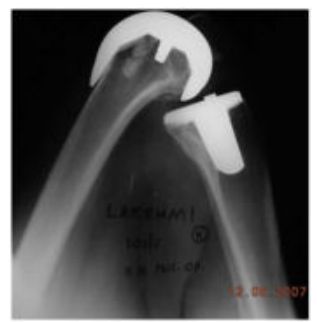

Case (pcl Sacrificed)


Postop Lateral View

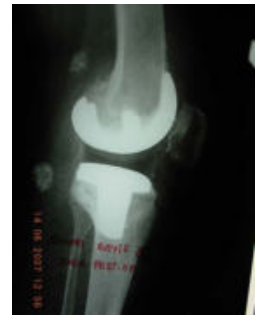

Postop Flexion
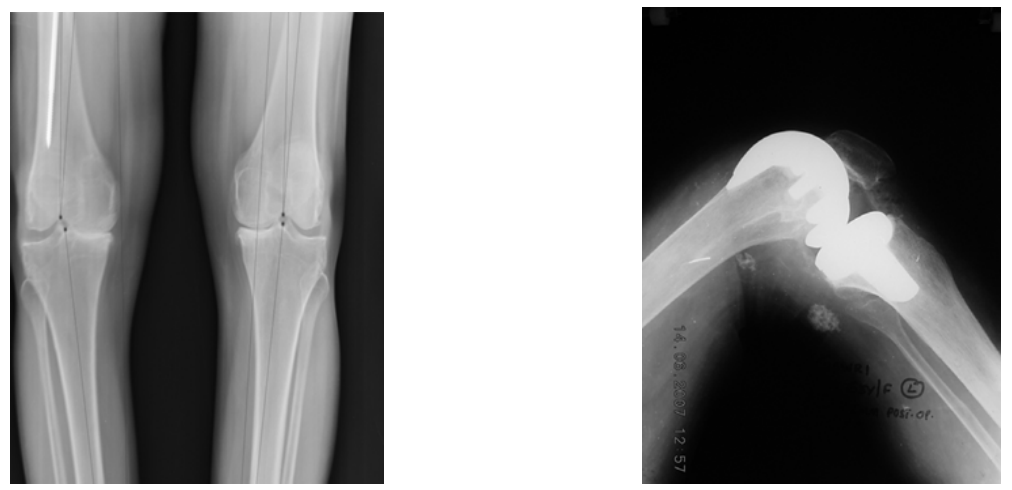

$\mid$ www.worldwidejournals.com $\mid$ 
Postop Extension

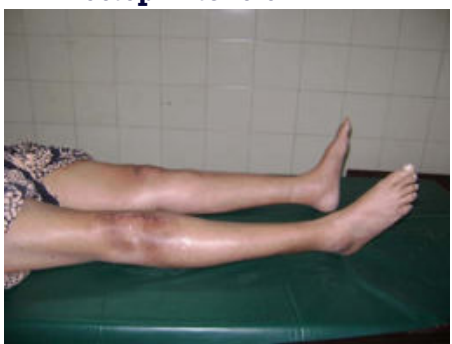

Postop Slr

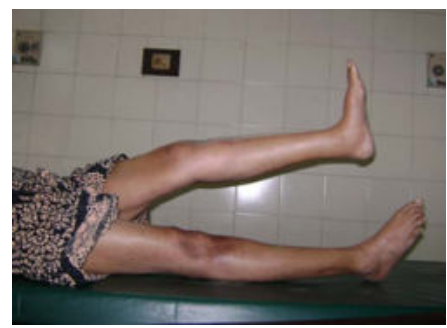

Postop Flexion

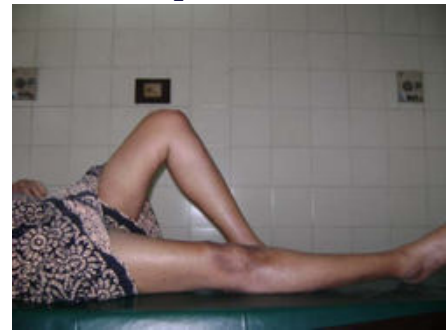

\section{v.CONCLUSION:}

In our short term analysis of this comparative study good results were obtained in both posterior cruciate retaining Total Knee Arthroplasty and posterior cruciate sacrificing Total Knee Arthroplasty.

However,Posterior cruciate cruciate retaining Total Knee Arthroplasty had a marginally better outcome than the posterior cruciate sacrificing Total Knee Arthroplasty but it needs a long term analysis.

\section{REFERENCES}

1. Merrill A. Ritter, Michael E.Berend, John B.Meding and Brain M.Crites. Long term followup of anatomic graduated components posterior cruciate retaining total knee replacement. Clin Orthop. 2001;388,pp.51-57.

2. Scott RD, Volatile TB. Twelve years experience with posterior retaining total knee arthroplasty. Clin Orthop 1986;205:100-7.

3. Insall JN.presidential address to the knee society. Choices and compromises in total knee arthroplasty. Clin Orthop 1998;226:43-8.

4. Kleinbert FA,Bryk E,Evangelista J, Scott WN, Vigorita VJ histological comparison of posterior cruciate ligaments from arthritic and age matched knee specimens .J Arhtroplasty 1996; 11:726-31.

5. Freeman MAR, Insall N,Besser W, Walker PS, Hallel T. Excision of the cruciate ligaments in total knee replacement. Clin Orthop 1977;126:209-12.

6. Matuda S, Whiteside LA, White SE,McCarthy DS. Knee kinematics of posterior cruciate ligament sacrificed total knee arthroplasty. Clin Orthop 1997; 341 : 257-66.

7. Dennis DA, Komisteck RD, Hoff WA, Gabriel SM. In vivo knee kinematics derived using an inverse perspective technique. Clin Orthop 1996;331: 10717 .

8. Scott RD, Thornhill TS. Posterior cruciate supplementing total knee replacement using conforming inserts and cruciate recession; effect on range of motion and radiolucent lines. Clin Orthop 1994;309: 146-9.

9. John N. Insall, Lawrence D. Dorr, Richard D. Scott, and W. Norman Scott: Rationale of the knee society clinical rating system. Clin Orthop. 1989 Nov; (248): 13-14.

10. Kellgren JH, Lawrence JS. Radiological assessment of osteoarthrosis. Ann Rheum Dis 1957; 16:494-502.

11. Jensen LK, Eenberg W: occupation as a risk factor for knee disorders. Scand J Work Environ Health. 1996 Jun; 22 (3): 165-75.

12. coggon D, Croft P, Kellingray S, Barrett D, McLaren M, Cooper C: occupational physical activities and osteoarthritis of the knee. Arthritis Rheum. 2000 Jul; 43 (7): 1443-9.

13. Jensen Lk. Knee-straining work activities, self reported knee disorders and radiographically determined knee oarthritis. Scand J Work Environ Health. 2005;31 Suppl 2:68-74.

14. Manninen P, Heliovaara M,Riihimaki H,Suoma-Iainen O. physical work load and the risk of severe knee osteoarthritis. Scand J Work Environ Health 2002 Feb;28(1):25-32.
15. Kuster M, Wood GA, Sakurai S, Blatter G. stress on the biomechanical femoral patellar joint in downhill walking a biomechanical study. Z Unfallchir Versicherungsmed.1993:86(3):178-83

16. Kujala Um, Kettunen J Paananen H, Aalto T, Battie MC, Impivaara O,Videman T, Sarna S. knee osteoarthritis in former runners, soccer players, weight lifters, and shooters. Arthritis Rheum. 1995 Apr; 38 (4):539-46.

17. McMillan G, Nicholas L, osteoarthritis and meniscus disorders of the knee as . 2005 Aug: 62 (8): 567 75. 